\title{
DOPPLER ECHOCARDIOGRAPHY IMAGING AS A USEFUL TOOL IN DETECTING RHEUMATIC SILENT CARDITIS
}

\author{
Bindu A ${ }^{1}$, Santhosh Jose ${ }^{2}$, Soumya Jose ${ }^{3}$
}

\section{HOW TO CITE THIS ARTICLE:}

Bindu A, Santhosh Jose, Soumya Jose. "Doppler Echocardiography Imaging as a useful Tool in Detecting Rheumatic Silent Carditis". Journal of Evolution of Medical and Dental Sciences 2014; Vol. 3, Issue 40, September 01; Page: 10146-10152, DOI: 10.14260/jemds/2014/3318

ABSTRACT: BACKGROUND: Rheumatic fever and rheumatic heart disease continue to be an important public health problem in developing countries. Doppler echocardiography is now widely used for early detection and recurrence of clinical evident carditis (CC) and silent (subclinical) carditis (SC). In this present study, we performed Doppler echocardiography in ARF children clinically diagnosed by the Jones criteria in order to compare its effectiveness in detecting single/multi-valvular lesions over clinical evaluation alone and they were followed with repeat examinations over a period of 6 months. METHODS: A total of 57 consecutive patients diagnosed with acute rheumatic fever were included in the study. The patients without clinical evidence but with echocardiographic findings of carditis were diagnosed as having subclinical carditis. RESULTS: Acute rheumatic fever was diagnosed in 57 patients, and 38 of these had carditis. Among the 38 clinically 25(65.8\%) patients were detected to have cardiac lesion. Echocardiography showed thirteen (34.2\%) more patients were affected with carditis. The prevalence of SC was $13(22.8 \%)$ among these 57 patients. 51patients were followed up for 6months, and 11 of those had SC. CONCLUSION: It is suggested that Doppler echocardiography be performed in all patients with suspected acute rheumatic fever for early detection of SC. Echocardiography should be used as a diagnostic criterion in order not to miss a diagnosis of SC.

KEYWORDS: Rheumatic fever, echocardiography, carditis

INTRODUCTION: Acute rheumatic fever is a non-supportive complication of group A beta haemolytic streptococcal sore throat (GABHS). It affects joints, heart, brain, skin and sub cutaneous tissue.(1) Except for heart, all other effects are reversible, needing only symptomatic treatment during the episodes. Cardiac complications are significant in the absence of prophylaxis and culminate into chronic and life threatening valvular diseases.(2) Between 1940 and 1983, the prevalence rate of rheumatic heart disease varied from 1.8 to 11 per 1000, and between 1984 and 1995 the rate varied from 1 to 5.4 per $1000 .[3]$

A large study conducted by the Christian Medical College and Hospital (Vellore, India) reported the prevalence of rheumatic heart disease among school-going children to be 0.68 per 1000.[3] The criteria for diagnosing acute rheumatic fever were first established by Duckett Jones in $1944,{ }^{[4]}$ but the guidelines have since undergone several revision. ${ }^{[5]}$ Common to each update, carditis has remained one of the major criteria for diagnosing acute rheumatic fever.

Cardiac involvement in acute rheumatic fever is traditionally investigated by auscultation. In most cases, this either leads to an under-diagnosis, whereby nearly half of the patients with established rheumatic heart disease don't receive antibiotic prophylaxis, or to an over-diagnosis, which can leads to unnecessary treatment with potentially harmful medications (including steroids). With the advent of cross sectional echocardiography and colour flow Doppler imaging, it has been 


\section{ORIGINAL ARTICLE}

claimed that mitral and aortic valve insufficiency can be detected in up to $90 \%$ of rheumatic fever patients who have no clinical evidence of carditis $(5,6)$.

Yet, echocardiography is not included as a criterion in the diagnosis of acute rheumatic fever. Pathophysiologically, repeated rheumatic carditis can result in subvalvular or valvular thickening before the development of leaflet retraction and thereby regurgitation. Thus, we have previously proposed that morphological changes of valves affected by the rheumatic inflammatory process, even before the development of pathological regurgitation, likely indicate subclinical rheumatic heart disease, which might benefit from identification and thence secondary prevention. ${ }^{(7)}$

In this present study, we performed Doppler echocardiography in acute rheumatic fever children clinically diagnosed by the Jones criteria in order to compare its effectiveness in detecting single/multi-valvular lesions over clinical evaluation alone and they were followed with repeat examinations over a period of 6 months.

METHODS: We performed clinical examination and recorded echocardiographic parameters in all the consecutively enrolled 57 children, who were diagnosed with acute rheumatic fever and attended or were admitted to the paediatrics department at the institute of maternal and child health, medical college Hospital, Calicut Kerala during the period from May 2004 to April 2007. Ethical committee of the institution has approved the study.

All patients were under 12 years of age, and had a clinical diagnosis of acute rheumatic fever, according to the modified Jones criteria. After obtaining written consent from caretakers, the subject children were enrolled into the study and subsequently evaluated. Demographic details, clinical features at presentation, and anthropometry (height, weight, and BMI) were recorded. Each case was examined in detail. Physical findings were recorded and a detailed cardiovascular examination was done for each case. Enrolled children underwent routine blood tests, antistreptolysin 0 titer, ECG, chest X- ray and echo-Doppler in the hospital setting.

Echo-Doppler was done by the same cardiologist for the uniformity Once the diagnosis of acute rheumatic fever was confirmed (by clinical and/or echo), treatment started according to standard guidelines (8). Twice daily penicillin prophylaxis advised.

All echocardiographic recordings were done at a single hospital centre and using the same machine. Results were interpreted by the same person to avoid inter observer variability; and was blinded to all clinical information.

STATISTICAL ANALYSIS: Chi-square test (x2 test) was used in the two groups to compare the categorical data. Probability values of $\mathrm{p}<0.05$ were considered significant. 


\section{RESULTS:}

\begin{tabular}{|l|c|}
\hline \multicolumn{1}{|c|}{ Total number of ARF patients } & $\mathbf{5 7}$ \\
\hline Sex & \\
\hline Male & $32(56)$ \\
\hline Female & $25(44)$ \\
\hline Age (year) (median (range)) & $11(3.5-12)$ \\
\hline First ARF episode & $50(88)$ \\
\hline Polyarthritis & $44(77)$ \\
\hline Carditis & $38(67)$ \\
\hline Chorea & $4(7)$ \\
\hline Erythema marginatum & 0 \\
\hline Subcutaneous nodules & 0 \\
\hline
\end{tabular}

Table 1 Baseline characteristics and major Jones criteria

The male: female ratio was $1.3: 1$ and the median age (range) was $11(3.5-12$ ) years. $82 \%$ patients belonged to the upper lower and lower middle class. Most cases were admitted during spring season.

\begin{tabular}{|c|c|c|c|c|}
\hline \multicolumn{3}{|c|}{} & \multicolumn{2}{c|}{ A=57 } \\
\hline \multirow{2}{*}{ Valve involvement } & \multicolumn{2}{|c|}{ At entry n-57 } & Follow up (6months) n-51 \\
\cline { 2 - 5 } & Clinical & Clinical + Echo & Clinical & Clinical Echo \\
\hline Mitral & 16 & 22 & 11 & 15 \\
\hline Aortic & 9 & 16 & 6 & 13 \\
\hline Tricuspid & 1 & 3 & 0 & 3 \\
\hline Total & $\mathbf{2 5}$ & $\mathbf{3 8}$ & $\mathbf{1 7}$ & $\mathbf{2 8}$ \\
\hline Normal & 19 & & 23 & \\
\hline Table 2. Clinical (auscultatory) and echo Doppler detection of valve lesions \\
in patients during the acute episode and during follow up
\end{tabular}

Out of total of 57 patients, 38 patients had carditis. Among the 38 clinically 25 (65.8\%) patients were detected to have cardiac lesion. Echocardiography showed thirteen (34.2\%) more patients were affected with credits. P0.01. Mitral involvement was there in 22 patients, 16 had aortic, and 3 had tricuspid involvement.

Among these 15 had multi valvular involvement. After 6 months follow up 11 patients were detected to have mitral valve affected clinically, echocardiography picked up mitral lesion in 4 more patients. 13 with aortic involvement, only 6 picked up clinically, 7 more by echocardiography. So among total of 28 patients with cordites 17 (60.7\%) had clinical credits and 11 (39.3\%) had subclinical cordites.

Among the various echocardiographic abnormalities in patients with cordites, the most common was thickened mitral valve, followed by mitral regurgitation, aortic regurgitation and tricuspid regurgitation 


\section{ORIGINAL ARTICLE}

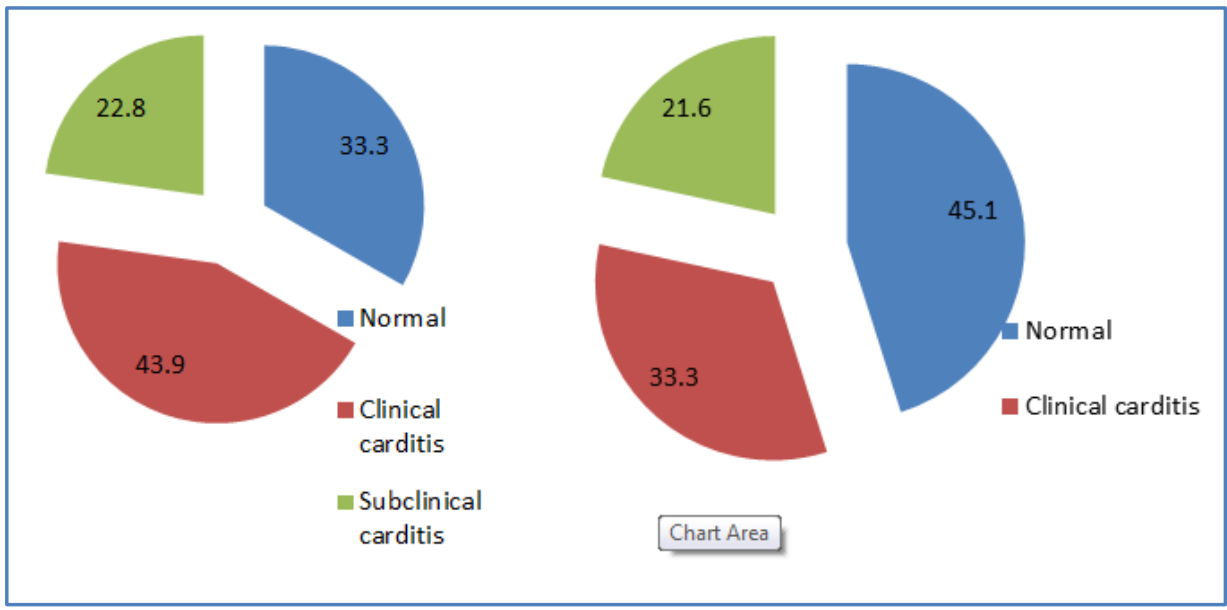

Fig. 1: Subclinical and clinical valve lesions in rheumatic fever patients at entry (A) and at 6months (B)

The above figure denotes patients who were found to have subclinical valve disease (subclinical), clinically evident or auscultatory disease (clinical), or no detectable valve lesions, either clinical or echocardiographic (no rheumatic heart disease) during the acute episode (fig. 1A) and after 6 months (fig. 1B) of follow up.

Among 57 cases, 51 cases came for follow up at 6months. 6 were lost follow up. The percentage of individuals with subclinical valve lesions, as well as those with clinical lesions, decreased with time. Out of 13 patients who had subclinical cordites at first visit, 9 patients had persistence of carditis. 3 had normal echo, 1 lost for follow up. Two patients, who initially had clinical cordites, follow up at 6 months revealed normal examination findings but echo showed thickened mitral valve. There was no significant differences after 6 months between clinical (65.8\% and 60.7\%) or subclinical (34.2\% and 39.3\%) valve lesions that persisted throughout follow up. p value was 0.94 . Discussion

Rheumatic fever and rheumatic heart disease continue to be an important public health problem in developing countries. According to the World Health Organisation, at least 15.6 million people have rheumatic heart disease, and 0.3 million out of 0.5 million individuals who acquire acute rheumatic fever every year develop rheumatic heart disease later.

In each year, 233, 000 deaths are directly attributable to acute rheumatic fever or rheumatic cardiac disease. Hence, detection of active rheumatic cordites is of great prognostic and therapeutic importance. This is currently based on the use of the Jones' criterions ${ }^{8,9}$. The diagnosis of carditis in acute rheumatic fever traditionally depends on characteristic auscultatory findings, like the detection of a new murmur which was not present earlier, cardiomegaly, congestive cardiac failure, and findings of a pericardial rub.

These auscultatory findings are unreliable in developing countries, where it is difficult to identify a new murmur from pre-existing murmur. Cardiomegaly could be due to any other cause, and not necessarily acute rheumatic fever ${ }^{10}$. Rheumatic carditis is almost always associated with valvitis. The valvitis constitutes the phenotypic feature of rheumatic carditis, hence echocardiographic documentation of valvar and subvalvar changes can be of significant help 10 . 


\section{ORIGINAL ARTICLE}

Doppler echocardiography is now widely used for early detection and recurrence of clinical evident carditis (CC) and silent (subclinical) carditis (SC).

The prognosis of such patients with acute rheumatic fever and subclinical valvitis is critical when determining the duration of antibiotic prophylaxis. Doppler echocardiographic evidence of trivial-to-mild valvular regurgitation is commonly observed in a normal population. ${ }^{11}$ Physiological leaks do not usually extend more than $1 \mathrm{~cm}$ beyond the valve co-optation point (where the two valves fit together when they close). Pathological regurgitation is seen as a substantial colour jet in two planes extending beyond valve leaflets lasting throughout the cardiac cycle with a well-defined, highvelocity spectral envelope. ${ }^{12}$

In the known outbreak of rheumatic fever in the USA, carditis was diagnosed by auscultation in 53 of the 74 patients (72\%) while Doppler echocardiography detected mitral regurgitation in an additional 14 patients (19\%).13 Similar findings have been reported by Folger and colleagues in the Middle East ${ }^{6}$ by Abernethy and associates in New Zealand. ${ }^{14}$ and by F E Figueroa et al. ${ }^{15}$ In the present study, at first visit, $65.8 \%$ had clinical evidence of carditis. By echo additional $34.2 \%$ detected to have carditis which was statistically significant.

This finding of subclinical carditis is important because they are prone for infective endocarditis and follow up for these patients also should be done under regular basis. In our study at 6 months of follow up, 39.3\% patients had subclinical carditis. There was no statistically significant difference in the persistence of carditis. This indicates that even subclinical cardiac lesions are also persisting and can lead to problems. The high prevalence of rheumatic heart disease in developing countries could be partly because of under-diagnosis of RF owing to a lack of facilities for echocardiography and thus inability to detect subclinical regurgitation.(16)

Limitations of our study are 1 . Small sample size as it was a single centre study. 2. Long term follow up at least for five years should be done to know sequelae of those who are having subclinical carditis. The role of echocardiography screening in diagnosing carditis has long been made clear, but its inclusion as a major criterion in the Jones criteria is still under debate.

Two main reasons for this debate are that misinterpretation of results may lead to either under- or over-diagnosis, and that echo has limited availability in developing countries on account of cost effectiveness (17). When strict criteria are adhered to, the finding of pathological regurgitation on echocardiography, even though subclinical, should be accepted as a major criterion in patients with acute rheumatic fever.

CONCLUSION: Doppler echocardiography should be performed in all patients with suspected acute rheumatic fever for early detection of SC. Echocardiography should be used as a diagnostic criterion in order not to miss a diagnosis of SC.

Abbreviation: ARF-acute rheumatic fever, CC -clinical cordites, SC - Subclinical cordites.

\section{REFERENCES:}

1. English P C. Rheumatic fever in America and Britain. A biological epidemiological and medical history. New Jersy: Rutgers University Press; 1999. p. 17-52. 


\section{ORIGINAL ARTICLE}

2. Sanyal SK, Berry AM, Duggal S, Hooja V Ghosh S. Sequelae of the initial attack of acute rheumatic fever in children from north india.A prospective 5 year follow up study. Circulation 1982; 65: 375-379.

3. Padmavati S (2001) Rheumatic heart disease: prevalence and preventive measures in the Indian subcontinent Keywords: rheumatic heart disease; rheumatic fever. Heart 86: 127-127.

4. Jones T (1944). The diagnosis of rheumatic fever. JAMA: The Journal of the American Medical Association 126: 481-484.

5. Ferrieri P (2002) Proceedings of the Jones Criteria workshop. Circulation 106: 2521-2523.

6. Folger GM, Hajar R, Robida A, et al. Occurrence of valvar heart disease in acute rheumatic fever without evident carditis: colour flow Doppler identification. Br Heart J 1992; 67: 434-8.

7. Marijon E, Ou P, Celermajer DS, Ferreira B, Mocumbi AO, Jani D, Paquet C, Jacob S, Sidi D, Jouven $\mathrm{X}$. Prevalence of rheumatic heart disease detected by echocardiographic screening. N Engl J Med. 2007; 357: 470-476.

8. Special writing group of the Committee on Rheumatic fever, Endocarditis and Kawasaki disease of the Council of Cardiovascular disease in the young of the American Heart Association. Guidelines for the diagnosis of rheumatic fever: Jones criteria: 1992 update. JAMA 1992; 268: 2069-2073.

9. Vijayalakshmi IB, Mithravinda J, Deva AN. The role of echocardiography in diagnosing carditis in the setting of acute rheumatic fever. Cardiol Young 2005; 15: 583-588.

10. Ishwarappa B. Vijayalakshmi, Rajan 0. The efficacy of echocardiographic criterions for the diagnosis of carditis in acute rheumatic fever. Cardiol Young 2008; 18: 586-592.

11. Shrestha BR, Baniya GB, Raut KB, Sharma S. Rheumatic fever in adults. J Nepal Med Assoc. 1996; 34: 236-41.

12. Saxena A. Diagnosis of rheumatic fever: current status of Jones criteria and role of echocardiography. Indian J Pediatr 2000; 67: 283-6.

13. Veasy LG, Wiedmeier SE, Orsmond GS, et al. Resurgence of acute rheumatic fever in the intermountain area of the United States. N Engl J Med 1987; 316: 421-7.

14. Abernethy M, Bass N, Sharpe N, et al. Doppler echocardiography and the early diagnosis of carditis in acute rheumatic fever. Aust N Z Med 1994; 24: 530-5.

15. Figueroa F.E, M Soledad Fernández, P Valdés etal. Prospective comparison of clinical and echocardiographic diagnosis of rheumatic carditis: long term follow up of patients with subclinical disease. Heart 2001; 85: 407-410.

16. Rayamajh A, Sharma D, Shakya U. Clinical, laboratory and echocardiographic profile of acute rheumatic fever in Nepali children. Annals of Tropical Paediatrics (2007) 27, 169-177.

17. Shivaram P, Ahmed M I. Doppler Echocardiography Imaging in Detecting Multi- Valvular Lesions: A Clinical Evaluation in Children with Acute Rheumatic Fever. PLOS ONE; 2013; 8 e74114. 


\section{ORIGINAL ARTICLE}

\section{AUTHORS:}

1. Bindu A.

2. Santhosh Jose

3. Soumya Jose

\section{PARTICULARS OF CONTRIBUTORS:}

1. Assistant Professor, Department of Paediatrics, MES Medical College, Palachode, Mallappuram, Kerala.

2. Associate Professor, Department of Paediatrics, MES Medical College, Palachode, Mallappuram, Kerala.

3. Assistant Professor, Department of Paediatrics, MES Medical College, Palachode, Mallappuram, Kerala.

\section{NAME ADDRESS EMAIL ID OF THE} CORRESPONDING AUTHOR:

Dr. Bindu A,

Assistant Professor,

Department of Pediatrics,

MES Medical College,

Palachode (P.0)-679338,

Mallappuram (Dist.), Kerala.

Email: binduathor@yahoo.com

Date of Submission: 06/08/2014.

Date of Peer Review: 07/08/2014.

Date of Acceptance: 25/08/2014.

Date of Publishing: 01/09/2014. 